\title{
Potential Luminosity Improvement for Low-Energy RHIC Operation with Electron Cooling
}

\author{
Alexei V. Fedotov ${ }^{*}$ \\ Brookhaven National Laboratory \\ Upton, NY 11973, USA \\ E-mail: fedotovebnl.gov
}

\section{for Low-Energy RHIC cooling working group}

There is a strong interest in heavy-ion RHIC collisions in the energy range below the present RHIC injection energy, which is termed "low-energy" operation. These collisions will help to answer one of the key questions in the field of QCD about the existence and location of a critical point on the QCD phase diagram. However, luminosity projections are relatively low for the lowest energy points of interest. Luminosity improvement can be provided with RHIC electron cooling at low beam energies. This report summarizes the expected luminosity improvements with electron cooling and various limitations.

5th International Workshop on Critical Point and Onset of Deconfinement CPOD 2009 Brookhaven National Laboratory, Long Island, New York, USA.

June 8-12, 2009

\footnotetext{
Speaker

Work supported by Brookhaven Science Associates, LLC under contract No. DE-AC02-98CH10886 with the U.S. Department of Energy.
} 


\section{Introduction}

The low-energy RHIC program with heavy ion collisions is motivated by a search for the QCD phase transition critical point [1-4]. There have been several short test runs during 20062008 RHIC operations to evaluate RHIC operational challenges for energies below present injection energy [5]. Beam lifetimes observed during the test runs were likely limited by machine nonlinearities. This performance limit can be improved with sufficient machine tuning. The next luminosity limitation comes from transverse and longitudinal Intra Beam Scattering (IBS), and ultimately from the space-charge limit. Detailed discussion of limiting beam dynamics effects can be found in Refs. [6-7].

Electron cooling of RHIC ion beams will increase the average integrated luminosity, and will provide longer stores for physics. If a critical point signature is found, a larger statistical sample (on the order of 50M events) will likely be requested to characterize it. RHIC lowenergy electron cooling will enable acquisition of this data in a reasonable period of time.

Our present estimate is that electron cooling can provide about 3-6 fold increase in average luminosity for the lowest energies of interest. Note that if hard space-charge limit is not reached, electron cooling can provide additional factors in luminosity improvement on top of the factor 3-6 quoted above [6].

\section{Luminosity limitations}

\subsection{Intra Beam Scattering}

IBS is one of the major effects contributing to RHIC heavy ion luminosity degradation, driving bunch length and transverse beam emittance growth. IBS-driven bunch length growth causes beam losses from the RF bucket. Figures 1-2 show results of simulations of ion beam dynamics using the BETACOOL program [8], for low-energy beam parameters in Table 1.

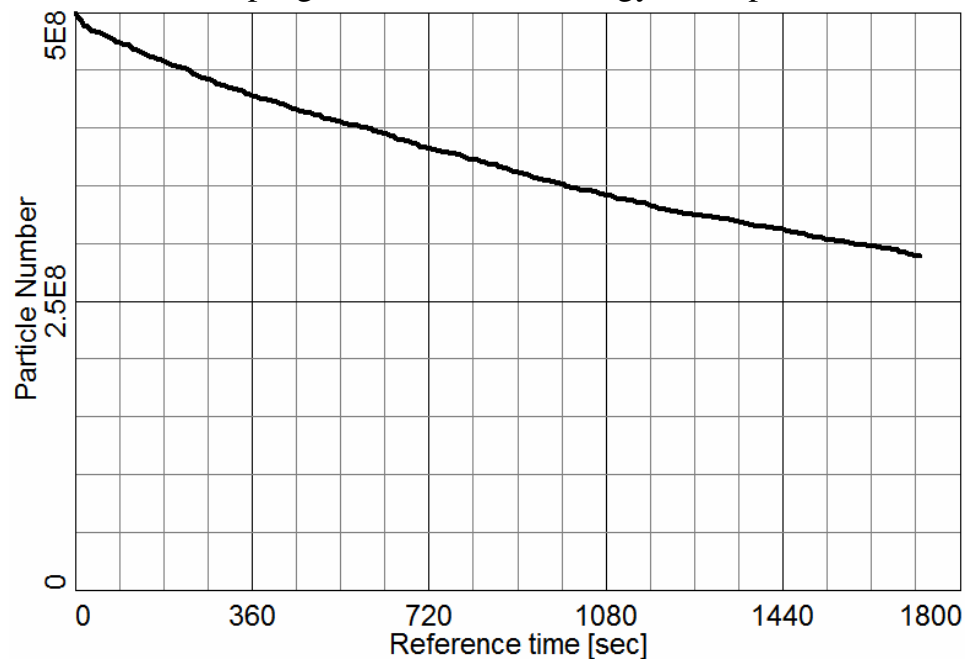

Figure 1: Simulated decrease of bunch intensity due to loss from the RF bucket caused by longitudinal IBS at the lowest energy point in the proposed RHIC energy scan with $\gamma=2.7$. 


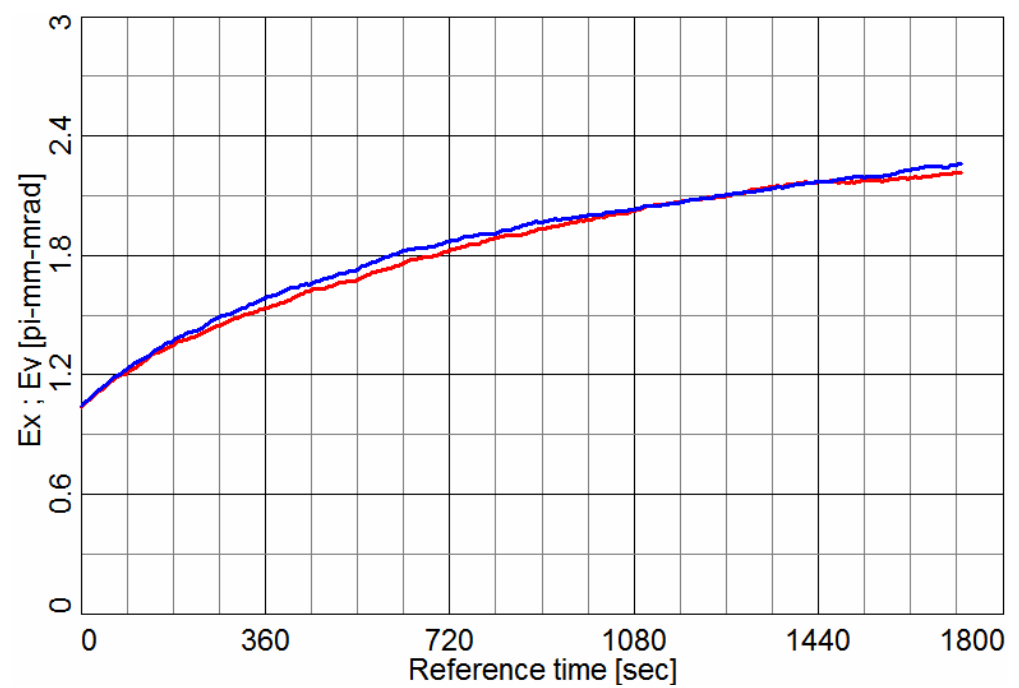

Figure 2: IBS-driven RMS un-normalized emittance growth at $\gamma=2.7$. Red curve - horizontal emittance. Blue curve - vertical emittance.

\begin{tabular}{|l|l|}
\hline$\gamma$ & 2.7 \\
\hline Number of ions per bunch, $10^{9}$ & 0.5 \\
\hline Initial transverse 95\% normalized emittance, mm-mrad & 15 \\
\hline RMS momentum spread & 0.0005 \\
\hline RF harmonic & 387 \\
\hline
\end{tabular}

Table 1: Parameters of RHIC low-energy gold ion beam used in IBS simulations for Figs. 1-2.

Beam luminosity depends on bunch intensity and beam emittance as

$$
L=\frac{N_{i}^{2}}{4 \pi \varepsilon \beta^{*}} F_{\text {coll }} f\left(\frac{\sigma_{s}}{\beta^{*}}\right) \text {, }
$$

where $N_{i}$ is the ion number per bunch, $\varepsilon$ is the transverse unnormalized rms emittance, $\beta^{*}$ is the beta function at the iteraction point (IP) and $\sigma_{s}$ is the rms value of the longitudinal beam size. $F_{\text {coll }}$ is the collision repetition frequency, which equals to the bunch revolution frequency $F_{\text {rev }}$ multiplied by the bunch number $N_{\text {bunches }}$. The factor $f\left(\sigma_{\mathcal{S}} \beta^{*}\right)$, which describes the "hourglass" effect, is close to unity when the longitudinal rms beam size is much less than the value of beta function at the IP, and decreases when $\sigma_{s}$ is increased.

At low-energy RHIC operation luminosity is decreased as a square of bunch intensity loss due to the longitudinal IBS and due to increase of the transverse emittance resulting from the transverse IBS. Both transverse and longitudinal IBS can be counteracted by a technique known as electron cooling. This allows one to keep the initial peak luminosity constant throughout the store without beam loss. In addition, the phase-space density of hadron beams can be further increased by providing stronger electron cooling, which results in further luminosity increase. 


\subsection{Space charge}

If IBS were the only limitation, one could achieve small hadron beam emittance and bunch length with the help of electron cooling, resulting in a dramatic luminosity increase. Unfortunately, the defining limitation is expected to be space charge at the lowest energy points in RHIC.

In circular accelerators, the figure of merit for space-charge effects is the shift of incoherent betatron oscillation frequencies. This is called the "space-charge tune shift". When the space-charge tune shift becomes significant, the spread of particle frequencies within the beam can overlap machine imperfection resonances, leading to large beam losses and poor lifetime. For machines where beam spends only tens of msec in high space-charge regime, and machines where the resonances are compensated, the tolerable space-charge tune shift can be as big as $\Delta \mathrm{Q}=0.2-0.5$. However the acceptable tune shifts are much smaller for long storage times. In some machines, lifetimes of few minutes were achieved with tune shifts of about 0.1 or higher. For RHIC, we are interested in lifetimes much longer than a few minutes. As a result, we take space-charge tune shift values of about 0.05 as a limit for our present estimate, which is somewhat conservative.

An even more interesting and unexplored effect is the interplay of both direct space-charge and beam-beam effects, which is the case when one wants to collide beams with significant space-charge tune shift. In such a case, large beam-beam parameter can excite resonances which will be crossed as a result of space-charge tune spread. We started to explore these effects in Accelerator Physics Experiments (APEX) in 2009 with proton beam and plan to continue next year with gold ion beams.

\subsection{Beam-beam}

The linear part of the tune shift due to interaction with a colliding bunch is called the "beam-beam" parameter. If the beam-beam parameter exceeds some limiting value one can have significant increase of emittance due to diffusion processes. In hadron machines, typical limiting values for the beam-beam parameter per single IP are 0.005-0.01.

For a round beam, the beam-beam parameter for hadrons is:

$$
\xi=\frac{Z^{2} r_{p}}{A} \frac{N_{i}}{4 \pi \beta^{2} \gamma \mathcal{E}} \frac{1+\beta^{2}}{2}
$$

where $r_{p}$ is proton classical radius, $A$ and $Z$ are the ion atomic and charge numbers, and $\gamma, \beta$ are relativistic factors. If the single bunch luminosity is limited by the beam-beam effect it can be expressed via $\xi$ as:

$$
L=\frac{A}{Z^{2} r_{p}} \frac{N_{i} c}{\beta^{*} C} \frac{2 \gamma \beta^{2}}{1+\beta^{2}} f\left(\frac{\sigma_{s}}{\beta^{*}}\right) \xi,
$$

where $\mathrm{C}$ is the ring circumference. If the luminosity is limited by the space-charge tune shift value $\Delta \mathrm{Q}$, then it can be expressed as:

$$
L=\frac{A}{Z^{2} r_{p}} \frac{N_{i} c}{\beta^{*}} \frac{\sqrt{2 \pi} \sigma_{s}}{C^{2}} \gamma^{3} \beta^{2} f\left(\frac{\sigma_{s}}{\beta^{*}}\right) \Delta Q,
$$


where the maximum (small-amplitude particles) space charge tune shift for a Gaussian transverse distribution can be estimated using the following formula:

$\Delta Q=-\frac{Z^{2} r_{p}}{A} \frac{N_{i}}{4 \pi \beta^{2} \gamma^{3} \varepsilon} \frac{C}{\sqrt{2 \pi} \sigma_{s}}$,

where we have assumed Gaussian longitudinal profile.

In the general case, the luminosity is limited by a minimum value from either Eq. (3) or (4). For the lowest energies in the proposed RHIC energy scan, the limitation comes from the space charge [6-7].

\section{Performance with cooling}

To provide electron cooling of hadron beams one needs to produce an electron beam with exactly the same kinetic energy as that of the hadrons. Thus providing cooling of ions with center-of-mass (c.m.) energies of 5-20 GeV/nucleon requires electron kinetic energies of 0.9-5 $\mathrm{MeV}$. Initially, we considered electron cooler with maximum energy of $2.8 \mathrm{MeV}$ which is needed to provide cooling for c.m. energies in the range of 5-12 GeV/nucleon. At higher energies luminosity is sufficiently high so that electron cooling is not really required, although it could significantly increase luminosity even further. However, our most recent cooling approach can provide cooling all the way up to c.m. energies of $20 \mathrm{GeV} /$ nucleon. Since this latter energy also corresponds to present injection energy of gold ions for typical high-energy RHIC program, the use of such cooler (with maximum electron energy of $5 \mathrm{MeV}$ ) can be beneficial for the RHIC high-energy program as well.

In this section some examples of luminosity improvement for lowest energies of interest are shown. Note that the bunch intensities at lowest energies may need to be reduced to respect the space-charge limit. As a result, the role of electron cooling for the lowest energy points is to counteract IBS: this prevents transverse emittance growth and intensity loss from the RF bucket due to the longitudinal IBS. As the energy is increased, space charge of the hadron beam becomes smaller (see Eq. (5)) which permits cooling of the transverse or longitudinal emittances of the hadron beams. This, in turn, allows us to reduce the beta function at the IP. Thus electron cooling provides a larger luminosity gain for higher energy points.

Figure 3 shows simulation of luminosity performance with and without electron cooling for $\gamma=2.67$. Simulations were performed for ion bunch intensity $\mathrm{N}_{\mathrm{i}}=0.5 \times 10^{9}$, initial $95 \%$ normalized emittance of $15 \mathrm{~mm}$ mrad, rms momentum spread $\sigma_{\mathrm{p}}=5 \times 10^{-4}$, and 56 bunches. There is an intensity loss as a result of the longitudinal IBS and particle loss from the RF bucket. There is also still a significant emittance increase due to the transverse IBS even for a reduced bunch intensity of $\mathrm{N}_{\mathrm{i}}=0.5 \times 10^{9}$ per bunch. This results in the rapid luminosity drop shown in Fig. 3 with black circles. The resulting store length becomes relatively short - one has to refill RHIC every 10-15 minutes. The transverse emittance will be kept constant, and the longitudinal IBS will be counteracted with electron cooling. As a result, electron cooling will provide long store times with relatively constant luminosity. The overall gain in average luminosity with electron cooling, taking into account needed time for refill between short stores without cooling, will be 
about a factor of 3. Larger luminosity gains may be possible if we can operate with spacecharge tune shifts larger than $\Delta \mathrm{Q}=0.05$. Operation with slightly larger tune shifts may be expected with the help of cooling.

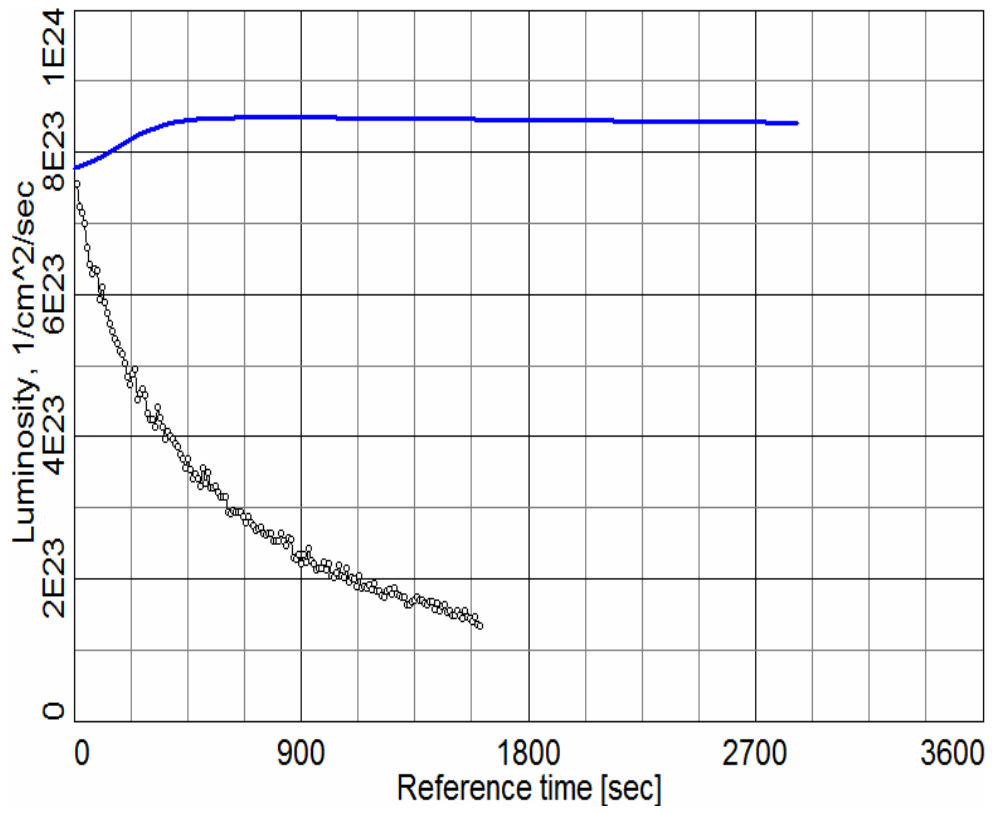

Figure 3: Simulation of "ideal" (no machine nonlinearities were included, just IBS and beam loss from RF bucket) luminosity with (blue line, upper curve) and without (black dots) electron cooling at $\gamma=2.7$ for 56 bunches.

Figure 4 shows a simulation of "ideal" luminosity performance with and without electron cooling for $\gamma=6.6$. Simulations were performed for ion bunch intensity $\mathrm{N}_{\mathrm{i}}=1 \times 10^{9}$, initial $95 \%$ normalized emittance of $15 \mathrm{~mm} \mathrm{mrad}$, and $\sigma_{\mathrm{p}}=5 \times 10^{-4}$. For these parameters we are not yet space-charge limited. In such a case, in addition to just counteracting IBS, electron cooling allows us to cool the transverse emittance to the space-charge limit, which in turn allows to decrease $\beta^{*}$ at the IP: the effect of this can be seen by the luminosity jump in Fig. 4 . Thereafter the luminosity remains constant. For the scenario shown in Fig. 4, electron cooling provides a factor of about 6 improvement in average luminosity. 


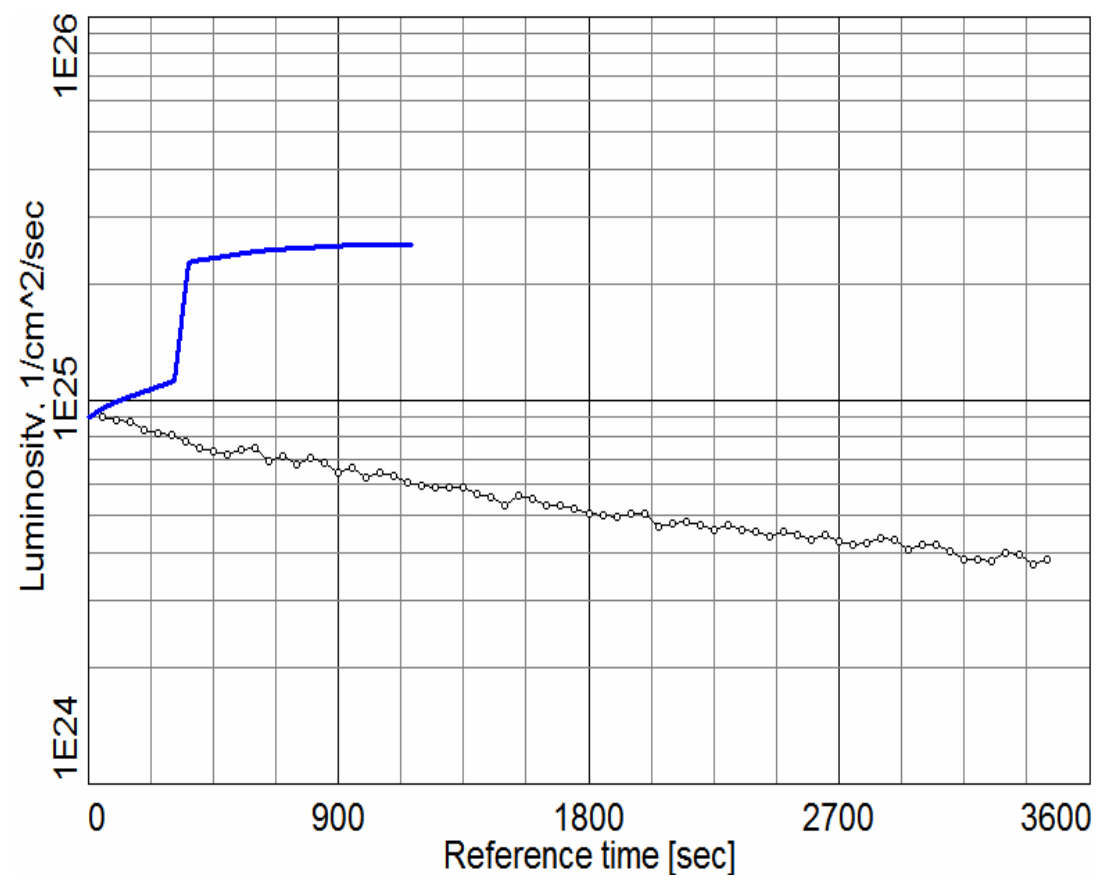

Figure 4: Simulation of luminosity with (blue line, upper curve) and without (black circles) electron cooling at $\gamma=6.6$.

To summarize, electron cooling offers longer stores for physics with about a factor of 3 improvement (additional factor might be possible if one could operate with slightly larger tune shifts with the help of cooling) in average luminosity for low-energy points $(\gamma=2.7-5)$ and about a factor of 6 or higher improvement for higher energy points $(\gamma>5)$. Further improvement in luminosity with cooling may be possible. In addition, electron cooling prevents growth of both transverse and longitudinal beam dimensions, limiting significant beam losses and associated radiation issues which may be an important issue for low-energy RHIC operations.

\section{Electron cooler considerations}

An electron beam with the needed energy and quality can be produced either using electrostatic or RF beam accelerator. Advantages and disadvantages of both approaches can be found in Ref. [6]. Our present baseline approach is based on DC electron beam produced with the FNAL Recycler's Pelletron. An evaluation of modifications to use the Recycler's Pelletron for RHIC is presently in progress. Details about electron cooler parameters and design are presented elsewhere [9].

\section{Acknowledgments}

We would like to thank I. Ben-Zvi, X. Chang, W. Fischer, D. Kayran, V. Litvinenko, A. Pendzick, E. Pozdeyev, T. Satogata, T. Roser and other members of Collider-Accelerator department at BNL for many useful discussions and help. We also thank A. Sidorin and A. 
Smirnov (JINR, Dubna) and S. Nagaitsev, A. Shemyakin, L. Prost (FNAL) and other members of FNAL's electron cooling group for providing very useful information.

\section{References}

[1] Proc. of Workshop "Can we discover QCD critical point at RHIC?" (March 2006) RIKEN Report BNL-75692-2006; http://www.bnl.gov/riken/QCDRhic.

[2] A. Cho, Science, V. 312, April 12, 2006, p 190

[3] G. Stephans, J. Phys. G: Nucl. Part. Phys. 32 (2006).

[4] M. Stephanov, K. Rajagopal, and E. Shuryak, Phys. Rev. Letters 81, p. 4816 (1998).

[5] T. Satogata et al., Proc. of PAC07 (Albuquerque, NM, 2007), p. 1877; Proc. of PAC09 (Vancouver, Canada, 2009) WE6PFP009; these proceedings.

[6] A. Fedotov et al., BNL C-AD Tech Note: C-A/AP/307 (2008).

[7] A. Fedotov et al., Proc. of HB2008 Workshop (Nashville, TN, 2008).

[8] BETACOOL code: http://lepta.jinr.ru; A. Sidorin et al., NIM A 558, p. 325 (2006).

[9] A. Fedotov et al., Proc. of COOL09 Workshop (Lanzhou, China, August 31-September 4, 2009). 